\title{
Correction to: Formal and Natural Proof: A Phenomenological Approach
}

\author{
Merlin Carl
}

\section{Correction to:}

Chapter 14 in: S. Centrone et al. (eds.), Reflections on the Foundations of Mathematics, Synthese Library 407, https://doi. org/10.1007/978-3-030-15655-8_14

The abstract of this chapter was initially published with error. The chapter has been updated with the corrected abstract as given below.

It is frequently claimed (see e.g. Rav, A critique of a formalist-mechanist version of the justification of arguments in mathematicians' proof practices. Philosophia Mathematica (III) 15:291-320, 2007) that the formalization of a mathematical proof requires a quality of understanding that subsumes all acts necessary for checking the proof and that, consequently, automatic proof checking cannot lead to an epistemic gain about a proof. We present a project developing what is sometimes called a 'fortified formalism' and argue, taking a phenomenological look at proof understanding, that proofs can be (and often are) given in a way that allows a formalization sufficient for producing an automatically checkable write-up, but does not subsume checking.

The updated version of this chapter can be found at https://doi.org/10.1007/978-3-030-15655-8_14 